\title{
IDENTIFYING PERTURBATIONS WHICH PRESERVE ASYMPTOTIC STABILITY ${ }^{1}$
}

\author{
AARON STRAUSS ${ }^{2}$ AND JAMES A. YORKE ${ }^{3}$
}

1. If the zero solution is uniform-asymptotically stable for the vector ordinary differential equation

$$
x^{\prime}=f(t, x)
$$

then it is also uniform-asymptotically stable for the perturbed equation

$$
y^{\prime}=f(t, y)+g(t, y)
$$

if $f$ satisfies a uniform Lipschitz condition and if $g$ is "sufficiently small." Such sufficiently small $g$ we will call permissible. This result is known and the proof is essentially the same as the proof that a Lipschitz, uniform-asymptotically stable system is totally stable [ 1 , p. 276]; namely, a positive definite, decrescent Lyapunov function $V$ exists for (E) satisfying $\dot{V}_{E}(t, x) \leqq-c(|x|)$ and $|\operatorname{grad} V(t, x)| \leqq b$. Therefore

$$
V_{P}(t, x)=\dot{V}_{E}(t, x)+\langle\operatorname{grad} V(t, x), g(t, x)\rangle \leqq-\frac{1}{2} c(|x|)
$$

if $|g(t, x)| \leqq c(|x|) / 2 b$. Thus an estimate on the size of permissible perturbations $g$ is provided in terms of a Lyapunov function associated with (E). If $f$ has further special properties, so might $V$. In this way Hahn $[1$, p. 282] proved the following:

Let $f_{x}(t, x)$ be continuous and bounded for $t \geqq 0$ and $|x| \leqq 1$. Suppose that for all real $c$ and some $k \geqq 1, f(t, c x)=c^{k} f(t, x)$. Then $g(t, x)=o\left(|x|^{k}\right)$ is permissible.

The purpose of this paper is to establish estimates on the size of permissible $g$ in terms of the rate of approach to zero of the solutions of $(E)$. Using these estimates we can prove Hahn's theorem without assuming that $f$ is differentiable.

2. Let $R^{n}$ denote Euclidean $n$-space. Let $\langle x, y\rangle$ denote the inner product of $x$ and $y$ in $R^{n}$, i.e., $\langle x, y\rangle=x_{1} y_{1}+\cdots+x_{n} y_{n}$. Let $|x|$

Presented to the Society, January 24, 1969 ; received by the editors November 13, 1968.

${ }^{1}$ Sponsored by the Mathematics Research Center, United States Army, Madison, Wisconsin, under Contract No. DA-31-124-ARO-D-462.

2 Work supported in part by an NSF Postdoctoral Fellowship and NSF grant GP6167 at the Department of Mathematics, University of Maryland.

3 Work supported in part by NSF grant GP-7846 at the Institute for Fluid Dynamics and Applied Mathematics, University of Maryland. 
$=\langle x, x\rangle^{1 / 2}$. Consider (E) and (P) where $f$ and $g$ map $[0, \infty) \times R^{n}$ continuously into $R^{n}$. Assume $f(t, 0)=g(t, 0)=0$. Thus for each $t_{0} \geqq 0$ and each $x_{0} \in R^{n}$, there is at least one solution $x\left(t ; t_{0}, x_{0}\right)$ of $(\mathrm{E})$ and at least one solution $y\left(t ; t_{0}, x_{0}\right)$ of $(\mathrm{P})$ through $\left(t_{0}, x_{0}\right)$ which are defined for $t$ in a neighborhood of $t_{0}$. (We do not assume that the solutions of $(\mathrm{E})$ or $(\mathrm{P})$ are uniquely determined by $\left(t_{0}, x_{0}\right)$.)

DEFINITION 2.1. The zero solution is uniform-asymptotically stable (UAS) for (E) if (i) for every $\epsilon>0$ there exists $\delta=\delta(\epsilon)>0$ such that $\left|x\left(t ; t_{0}, x_{0}\right)\right|<\epsilon$ for all $\left|x_{0}\right|<\delta$ and $t \geqq t_{0} \geqq 0$, and if (ii) there exists $\delta_{0}>0$ and for every $\eta>0$ there exists $T=T(\eta) \geqq 0$ such that $\left|x\left(t ; t_{0}, x_{0}\right)\right|$ $<\eta$ for $\left|x_{0}\right|<\delta_{0}, t_{0} \geqq 0$, and $t \geqq t_{0}+T$.

If the solutions of (E) are not uniquely determined by $\left(t_{0}, x_{0}\right)$, then the zero solution is UAS provided that (i) and (ii) above hold for all the solutions through $\left(t_{0}, x_{0}\right)$.

Following Hahn $[1$, p. 7] we say that a real-valued function $\phi(\cdot)$ belongs to class $K$ if, for some $r_{1}>0, \phi(\cdot)$ is continuous and strictly increasing on $\left[0, r_{1}\right]$ and $\phi(0)=0$.

3. We begin with a lemma which characterizes uniform-asymptotic stability in terms of certain auxiliary functions. These functions appear to be more useful for perturbation problems than those of Hahn [1, p. 8]; however, Hahn's functions seem more useful for converse theorems on Lyapunov functions [1, Chapter 6].

LEMMA 3.1. The zero solution of (E) is UAS if and only if there exist functions $\alpha(\cdot)$ and $\beta(\cdot)$ in $K$ and a positive function $\tau(\cdot)$ such that

$$
\alpha(\delta)<\delta \leqq \beta(\delta) \quad \text { for } 0<\delta<\delta_{0},
$$

and for all $\left|x_{0}\right| \leqq \delta<\delta_{0}, t_{0} \geqq 0$, and $t_{0} \leqq t \leqq t_{0}+\tau(\delta)$,

$$
\left|x\left(t ; t_{0}, x_{0}\right)\right| \leqq \beta(\delta) \text { and }\left|x\left(t_{0}+\tau(\delta) ; t_{0}, x_{0}\right)\right| \leqq \alpha(\delta) .
$$

Proof. If the zero solution is UAS, then $\beta(\cdot)$ exists by $[1$, p. 173]. Choose $\alpha(\delta)=\frac{1}{2} \delta$. Now we can take $\tau(\delta)=T\left(\frac{1}{2} \delta\right)$ by Definition 2.1.

Conversely, suppose (3.1) and (3.2) hold. Let $\epsilon>0$. Let $\delta=\delta(\epsilon)$ exist so that $0<\delta<\delta_{0}$ and $\beta(\delta)<\epsilon$. Let $\left|x_{0}\right| \leqq \delta$ and $t_{0} \geqq 0$. Then

$$
\left|x\left(t_{0}+\tau(\delta): t_{0}, x_{0}\right)\right| \leqq \alpha(\delta)<\delta .
$$

Therefore (3.2) holds with $t_{0}$ replaced by $t_{0}+\tau(\delta)$ and $x_{0}$ replaced by $x\left(t_{0}+\tau(\delta) ; t_{0}, x_{0}\right)$. Thus

$$
\left|x\left(t_{0}+2 \tau(\delta) ; t_{0}, x_{0}\right)\right| \leqq \alpha(\delta)<\delta ;
$$

hence (3.2) holds with $t_{0}$ replaced by $t_{0}+2 \tau(\delta)$. By induction 
$\left|x\left(t ; t_{0}, x_{0}\right)\right| \leqq \beta(\delta)<\epsilon$ for all $t \geqq t_{0}$. Thus (i) of Definition 2.1 holds.

Now choose $\delta_{n}=\alpha\left(\delta_{n-1}\right)$ and $t_{n}=\tau\left(\delta_{n-1}\right)+t_{n-1}$ for each $n=1,2, \cdots$. Since $\left\{\delta_{n}\right\}$ is a decreasing sequence of positive numbers, there exists $\vartheta \geqq 0$ such that $\delta_{n} \rightarrow \vartheta$ as $n \rightarrow \infty$. But $\delta_{n}-\alpha\left(\delta_{n}\right) \rightarrow \vartheta-\alpha(\vartheta)$ and $\delta_{n}-\alpha\left(\delta_{n}\right)$ $=\delta_{n}-\delta_{n+1} \rightarrow 0$. Hence $\alpha(\vartheta)=\vartheta$. By (3.1), $\vartheta=0$. Let $\eta>0$. Choose $N=N(\eta)$ so large that $\delta_{N}<\delta(\eta)$, where $\delta(\eta)$ comes from (i) of Definition 2.1. Consider any solution $\bar{x}\left(\cdot ; t_{0}, x_{0}\right)$ through $\left(t_{0}, x_{0}\right)$. Then

$$
\left|\bar{x}\left(t_{1} ; t_{0}, x_{0}\right)\right|=\left|\bar{x}\left(t_{0}+\tau\left(\delta_{0}\right) ; t_{0}, x_{0}\right)\right| \leqq \alpha\left(\delta_{0}\right)=\delta_{1} .
$$

Therefore, for some solution $x\left(\cdot ; t_{1}, \bar{x}\left(t_{1} ; t_{0}, x_{0}\right)\right)$,

$$
\left|\bar{x}\left(t_{2} ; t_{0}, x_{0}\right)\right|=\left|x\left(t_{2} ; t_{1}, \bar{x}\left(t_{1} ; t_{0}, x_{0}\right)\right)\right| \leqq \alpha\left(\delta_{1}\right)=\delta_{2} .
$$

By repeating this argument, we have that

$$
\left|\bar{x}\left(t_{N} ; t_{0}, x_{0}\right)\right|=\left|x\left(t_{N} ; t_{N-1}, \bar{x}\left(t_{N-1} ; t_{0}, x_{0}\right)\right)\right| \leqq \alpha\left(\delta_{N-1}\right)=\delta_{N} .
$$

Since $\delta_{N}<\delta(\eta)$, it follows that

$$
\left|\bar{x}\left(t ; t_{0}, x_{0}\right)\right|=\left|x\left(t ; t_{N}, \bar{x}\left(t_{N} ; t_{0}, x_{0}\right)\right)\right|<\eta
$$

for all $t \geqq t_{N}=t_{0}+T(\eta)$ for some solution $x\left(\cdot ; t_{N}, \bar{x}\left(t_{N} ; t_{0}, x_{0}\right)\right)$, where

$$
T(\eta)=\tau\left[\alpha^{(N-1)}\left(\delta_{0}\right)\right]+\tau\left[\alpha^{(N-2)}\left(\delta_{0}\right)\right]+\cdots+\tau\left[\delta_{0}\right] .
$$

Thus (ii) of Definition 2.1 holds. Hence the zero solution is UAS and Lemma 3.1 is proved.

We now restrict $f$ somewhat and prove a result concerning the distance of a solution of $(\mathrm{P})$ from one of (E). A similar result appears also in [2, Lemma 5.1].

Lemma 3.2. Suppose that for some $\gamma$ in the class $K$, some $L>0$, some $r>0$, and all $|x| \leqq r,|y| \leqq r$, and $t \geqq 0$, we have

$$
\begin{gathered}
\langle x-y, f(t, x)-f(t, y)\rangle \leqq L|x-y|^{2}, \\
|g(t, x)| \leqq \gamma(|x|) .
\end{gathered}
$$

Let $u>0, t_{0} \geqq 0$, and let $\left|x\left(t ; t_{0}, x_{0}\right)\right| \leqq r$ and $\left|y\left(t ; t_{0}, x_{0}\right)\right| \leqq r$ for $t_{0} \leqq t \leqq t_{0}+u$. Then for all $t_{0} \leqq t \leqq t_{0}+u$,

$$
\left|x\left(t ; t_{0}, x_{0}\right)-y\left(t ; t_{0}, x_{0}\right)\right| \leqq 2 \gamma(r) u e^{2 L u} \text {. }
$$

REMARK. If $f$ satisfies a uniform Lipschitz condition, i.e., $\mid f(t, x)$ $-f(t, y)|\leqq L| x-y \mid$ for all $t \geqq 0,|x| \leqq r$, and $|y| \leqq r$, then $f$ satisfies (3.3). Of course the converse is false, e.g. $f(t, x)=-t x^{3}$. If $f$ satisfies (3.3), then solutions of $(\mathrm{E})$ are uniquely determined by $\left(t_{0}, x_{0}\right)$ for $t>t_{0}$ but not necessarily for $t<t_{0}$. Even in this case solutions of $(\mathrm{P})$ need not be uniquely determined for $t>t_{0}$. 
Proof. Let $x(t)=x\left(t ; t_{0}, x_{0}\right)$ and $y(t)=y\left(t ; t_{0}, x_{0}\right)$. Define $\lambda=\sup$ $|x(t)-y(t)|$ for $t_{0} \leqq t \leqq t_{0}+u$. Then

$$
\begin{aligned}
\left\langle x^{\prime}(t)-y^{\prime}(t), x(t)-y(t)\right\rangle= & \langle x(t)-y(t), f(t, x(t))-f(t, y(t))\rangle \\
& -\langle x(t)-y(t), g(t, y(t))\rangle
\end{aligned}
$$

hence

$$
|x(t)-y(t)|^{2} \leqq 2 \lambda \gamma(r) u+\int_{t_{0}}^{t} 2 L|x(s)-y(s)|^{2} d s .
$$

By Gronwall's inequality

$$
|x(t)-y(t)|^{2} \leqq 2 \lambda \gamma(r) u e^{2 L u}
$$

for all $t_{0} \leqq t \leqq t_{0}+u$. Therefore $\lambda^{2} \leqq 2 \lambda \gamma(r) u e^{2 L u}$ from which the result follows.

4. Our main result says that if $f$ satisfies (3.3) and if the zero solution is UAS for (E) with corresponding $\alpha_{E}(\cdot), \beta_{E}(\cdot)$, and $\tau(\cdot)$, then by choosing appropriate larger $\alpha_{P}(\cdot)$ and $\beta_{P}(\cdot)$, there will be room enough to perturb (E) by certain functions $g$ and still have that the zero solution is UAS, but with corresponding $\alpha_{P}(\cdot), \beta_{P}(\cdot)$ and the same $\tau(\cdot)$.

THEOREM 4.1. Let $f$ satisfy (3.3). Let the zero solution of $(\mathrm{E})$ be $U A S$ with corresponding $\alpha_{E}(\cdot), \beta_{E}(\cdot)$, and $\tau(\cdot)$. Suppose there exist $\alpha_{P}(\cdot), \beta_{P}(\cdot)$, and $\gamma(\cdot)$ in the class $K$ such that for some $r>0$ and all $0<\delta \leqq r$, we have

$$
\alpha_{E}(\delta)<\alpha_{P}(\delta)<\delta \leqq \beta_{E}(\delta)<\beta_{P}(\delta),
$$

Then if $|g(t, x)| \leqq \gamma(|x|)$ for $t \geqq 0$ and $|x| \leqq r$, the zero solution of $(\mathrm{P})$ is $U A S$ with corresponding $\alpha_{P}(\cdot), \beta_{P}(\cdot)$, and $\tau(\cdot)$.

REMARKS. Note that the right-hand side of (4.2) is positive because of (4.1). Since $\beta_{P}(\cdot)$ is strictly increasing, $\gamma(\cdot)$ is well-defined by (4.2). Observe the structure of (4.2): the bound $\gamma(\cdot)$ for $g$ depends on the choices of $\beta_{P}(\cdot)$ and $\alpha_{P}(\cdot)$. To make $\gamma(\cdot)$ larger, one must take $\alpha_{P}(\cdot)$ closer to the identity function and thus obtain a slower approach to zero of the solutions of $(\mathrm{P})$. Actually, since $\alpha_{E}(\cdot), \beta_{E}(\cdot)$, and $\tau(\cdot)$ are not uniquely determined, some manipulating of these might result in better estimates for $\gamma(\cdot)$. This can be complicated because, for example, decreasing $\alpha_{E}(\cdot)$ would seem to force the increasing of $\tau(\cdot)$ which might make the right-hand side of (4.2) even smaller. The difficult but important problem of juggling all these scalar functions 
in order to obtain the best estimate of $\gamma(\cdot)$ from (4.2) has not been solved as yet. In some cases, it seems helpful to choose $\alpha_{E}(\cdot)$ in such a way that $\tau(\cdot)$ is constant (see the proof of Theorem 5.1). Example 8.2 of [2] shows that Theorem 4.1 need not hold if $f$ does not satisfy (3.3).

Proof. Let $\left|x_{0}\right|<\delta \leqq r$ and $t_{0} \geqq 0$. Let $x(\cdot)$ and $y(\cdot)$ be solutions of (E) and $(\mathrm{P})$, respectively, through $\left(t_{0}, x_{0}\right)$. For as long as $|y(t)| \leqq \beta_{P}(\delta)$ on the interval $t_{0} \leqq t \leqq t_{0}+\tau(\delta)$, we have

$$
\begin{aligned}
|y(t)| & \leqq|x(t)|+|y(t)-x(t)| \\
& \leqq \beta_{E}(\delta)+2 \gamma\left(\beta_{P}(\delta)\right) \tau(\delta) e^{2 L \tau(\delta)}<\beta_{P}(\delta) .
\end{aligned}
$$

Thus $|y(t)|<\beta_{P}(\delta)$ for $t_{0} \leqq t \leqq t_{0}+\tau(\delta)$. Also

$$
\begin{aligned}
\left|y\left(t_{0}+\tau(\delta)\right)\right| & \leqq\left|x\left(t_{0}+\tau(\delta)\right)\right|+\left|y\left(t_{0}+\tau(\delta)\right)-x\left(t_{0}+\tau(\delta)\right)\right| \\
& \leqq \alpha_{E}(\delta)+2 \gamma\left(\beta_{P}(\delta)\right) \tau(\delta) e^{2 L \tau(\delta)}<\alpha_{P}(\delta) .
\end{aligned}
$$

By Lemma 3.1, the zero solution is UAS for (P). This completes the proof.

5. We now apply Theorem 4.1 to obtain

THEOREM 5.1 Let $f$ satisfy (3.3) and for all real $c$ and some $k \geqq 1$ let

$$
f(t, c x)=c^{k} f(t, x) \text {. }
$$

Let the zero solution of $(\mathrm{E})$ be UAS. Then if $g(t, x)=o\left(|x|^{k}\right)$, the zero solution of $(\mathrm{P})$ is $U A S$.

REMARK. Hahn [1, p. 282] proved this result by using Lyapunov functions and under the additional assumption that $f$ has continuous first partial derivatives with respect to $x$ which are uniformly bounded with respect to $t$. Note that if $f$ is linear in $x$, then $f$ satisfies (5.1) with $k=1$.

Proof. First, assume $k=1$. Then $[1$, p. 280] there exist $a \geqq 1$ and $b>0$ such that

$$
\left|x\left(t ; t_{0}, x_{0}\right)\right| \leqq a\left|x_{0}\right| \exp \left[-b\left(t-t_{0}\right)\right]
$$

for all $t \geqq t_{0} \geqq 0$. Thus we may choose $\beta_{E}(\delta)=a \delta, \alpha_{E}(\delta)=\frac{1}{2} \delta$, and $\tau(\delta) \equiv \tau=b^{-1} \log 2 a$. Let $\beta_{P}(\delta)=(a+1) \delta$ and $\alpha_{P}(\delta)=3 \delta / 4$. Then the right-hand side of (4.2) is a linear function of $\delta$. Thus if $g(t, x)=o(|x|)$, (4.2) will be satisfied for sufficiently small $\delta$. that

Now let $k>1$. Then $[1$, p. 279-80] there exist $a>0$ and $b>0$ such

$$
\left|x\left(t ; t_{0}, x_{0}\right)\right| \leqq\left(a\left|x_{0}\right|^{1-k}+b\left(t-t_{0}\right)\right)^{1 /(1-k)}
$$


for $t \geqq t_{0} \geqq 0$ and there exist $c>0$ and $T>0$ such that

$$
\left|x\left(t ; t_{0}, x_{0}\right)\right| \leqq\left(\left|x_{0}\right|^{1-k}+c\left(t-t_{0}\right)\right)^{1 /(1-k)}
$$

for $t_{0} \geqq 0$ and $t \geqq t_{0}+T$. Thus we may choose $\beta_{E}(\delta)=a_{1} \delta, \alpha_{E}(\delta)$ $=\delta\left(1-\delta^{k-1}\right)^{1 /(k-1)}$, and

$$
\tau(\delta) \equiv \tau=2^{k-1}\left(c\left(2^{k-1}-1\right)\right)^{-1}+T,
$$

where $a_{1}=a^{1 /(1-k)}$. Then if $\left|x_{0}\right| \leqq \delta \leqq \frac{1}{2}, \tau \geqq\left(c\left(1-\delta^{k-1}\right)\right)^{-1}$; hence

$$
\left|x\left(t_{0}+\tau ; t_{0}, x_{0}\right)\right| \leqq \alpha_{E}(\delta) .
$$

Let $\beta_{P}(\delta)=\left(a_{1}+1\right) \delta$ and $\alpha_{P}(\delta)=\frac{1}{2}\left(\delta+\alpha_{E}(\delta)\right)$. Then the right-hand side of (4.2) becomes $q \delta\left[1-\left(1-\delta^{k-1}\right)^{1 /(k-1)}\right]$ for some constant $q>0$. If this expression is divided by $\left[\beta_{P}(\delta)\right]^{k}$, its limit as $\delta \rightarrow 0$ is, using L'Hospital's rule, a positive constant. Thus (4.2) will be satisfied for sufficiently small $\delta$ provided that $\gamma\left(\beta_{P}(\delta)\right) /\left[\beta_{P}(\delta)\right]^{k} \rightarrow 0$ as $\delta \rightarrow 0$, i.e., provided that $g(t, x)=o\left(|x|^{k}\right)$. This completes the proof.

\section{REFERENCES}

1. W. Hahn, Stability of motion, Springer-Verlag, New York, 1967.

2. A. Strauss and J. A. Yorke, Perturbing uniform asymptotically stable nonlinear systems, J. Differential Equations (to appear).

UNIVERSity OF Wisconsin, MADISON AND UNIVERSITY OF MARYLAND 